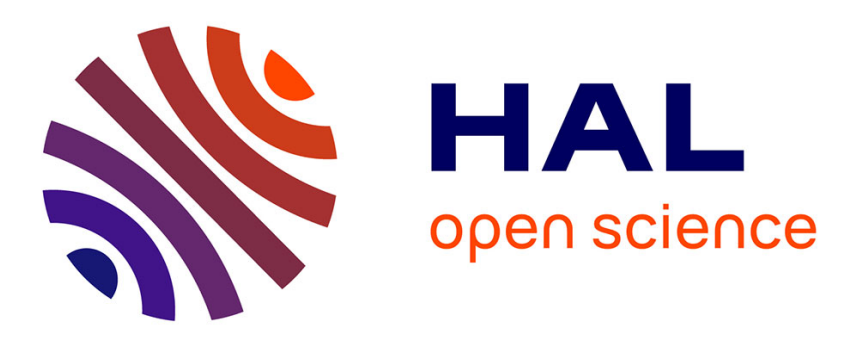

\title{
Capillary imbibition of aqueous foams by miscible and nonmiscible liquids
}

Rémy Mensire, Keyvan Piroird, Elise Lorenceau

\section{To cite this version:}

Rémy Mensire, Keyvan Piroird, Elise Lorenceau. Capillary imbibition of aqueous foams by miscible and nonmiscible liquids. Physical Review E : Statistical, Nonlinear, and Soft Matter Physics, 2015, 92 (5), 10.1103/PhysRevE.92.053014 . hal-01262037

\section{HAL Id: hal-01262037 \\ https://hal-enpc.archives-ouvertes.fr/hal-01262037}

Submitted on 26 Jan 2016

HAL is a multi-disciplinary open access archive for the deposit and dissemination of scientific research documents, whether they are published or not. The documents may come from teaching and research institutions in France or abroad, or from public or private research centers.
L'archive ouverte pluridisciplinaire HAL, est destinée au dépôt et à la diffusion de documents scientifiques de niveau recherche, publiés ou non, émanant des établissements d'enseignement et de recherche français ou étrangers, des laboratoires publics ou privés. 


\title{
Capillary imbibition of aqueous foams by miscible and non-miscible liquids
}

\author{
Rémy Mensire, ${ }^{1}$ Keyvan Piroird, ${ }^{1}$ and Elise Lorenceau ${ }^{1}$ \\ ${ }^{1}$ Université Paris-Est, Laboratoire Navier (UMR 8205 CNRS, ENPC ParisTech, \\ IFSTTAR), 2 allée Kepler, 77420 Champs-sur-Marne, France
}

\begin{abstract}
When put in contact with a large liquid drop, dry foams wick owing to surface-tension-driven flows until reaching equilibrium. This work is devoted to the dynamics of this imbibition process. We both consider imbibition of wetting or non-wetting liquid, either by putting the dry foam into contact with the foaming solution that constitutes the foam or with organic oils. Indeed, with the appropriate choice of surfactants, oil spontaneously invades the liquid network of the foam without damaging it. Our experiments show an early-time dynamics in $t^{1 / 2}$ followed by a late-time dynamics in $t^{1 / 4}$. These features, which differ from theoretical works predicting a late-time $t^{1 / 3}$ dynamics, are rationalized considering the influence of the initial liquid fraction of the foam in the driving capillary force and the impact of gravity through the capillary-gravity equilibrium.
\end{abstract}




\section{INTRODUCTION}

The earliest studies on impregnation of a single vertical capillary tube from an infinite reservoir date back to the 1700's with the pioneer works of Jurin, Newton and later on, Laplace. Subsequent studies have shown that capillary rise exhibits several dynamical regimes, such as the famous Lucas-Washburn $t^{1 / 2}$ scaling behavior for early times $[1,2]$.

In such a model geometry, the radius of the tube is the only length scale of the system and both sets the amplitude of the capillary pressure drop and the viscous dissipation. In homogeneous porous media with more complex geometries such as packed beds of granular beads, dry soils or paper, various dynamics have been experimentally reported. The imbibition dynamics of porous media characterized by a unique length scale, is logically described by the $t^{1 / 2}$ law $[2-5]$. Yet, other dynamics also occur in more complex media, such as deformable or heterogeneous media, where a unique length scale can not be defined unequivocally. The capillary rise of water in column of glass beads follows a $t^{1 / 4}$ regime for late times due to micronic heterogeneities of the glass surface $[4,6,7]$ and deformable sponges wick with an unexplained $t^{1 / 4}$ dynamics at late times [7]. A similar $t^{1 / 4}$ dynamics is also observed in heterogeneous media with gradients of pore size [8] and the imbibition between flexible boundaries, which results from an interplay between capillary, gravity and elasticity of the boundaries, exhibits different regimes [9].

In this context, dry aqueous foams, which provide perfectly smooth boundary conditions for the liquid, are interesting examples of complex deformable porous media. In those porous systems, the liquid is distributed between the thin films, the slender liquid-filled channels, called Plateau borders and the nodes as depicted in Figure 1-b) and c). When a dry liquid foam is put into contact at its bottom with its constitutive foaming solution, the liquid spontaneously rises within the liquid network following a $t^{1 / 2}$ law as experimentally observed in microgravity conditions[10-12]. On Earth, theoretical predictions concerning semi-infinite foams with an initial liquid fraction equal to zero report that the capillary rise continues indefinitely despite gravity, following a $t^{1 / 3}$ law [13] that recalls capillary rise observed in corners [14-16]. However, to the best of our knowledge, this scaling law has never been observed experimentally. 
In this letter, we therefore consider the imbibition dynamics of dry aqueous foams. We use both miscible liquid or an immiscible oil. Indeed, the remarkable imbibition ability of a liquid foam also holds when it is put into contact with an immiscible liquid, provided that it is not a defoaming agent for the foam, as recently shown at the scale of a single Plateau border or in confined geometries [17-19].

The foaming solution we use is described in $[17,20,21]$ and consists in a mixture of different components (cocoamidopropyl betaine, sodiumlaurylethyl sulfate, myristic acid and glycerol). It provides rigid boundary conditions of the air/foaming interfaces due to the presence of the fatty myristic acid [20, 21]. The air/foaming solution surface tension, viscosity and density of this mixture are $\gamma_{\mathrm{aw}}=23.7 \pm 1 \mathrm{mN} / \mathrm{m}$, $\eta_{F}=1.4 \mathrm{mPa} . \mathrm{s}$ and $\rho_{F}=1026 \mathrm{~kg} / \mathrm{m}^{3}$. The liquids of viscosity and density $\eta$ and $\rho$, are either miscible or immiscible with the foaming solution. Aqueous miscible solutions with viscosity $\eta$ ranging from 1.4 to $100 \mathrm{mPa} . \mathrm{s}$, are obtained by adding glycerol to the foaming solution. To maintain the architecture of the foam unaltered during the imbibition of immiscible fluids, we use two different oils which do not exhibit any defoaming activity. This requires that the oil droplets trapped within the foaming liquid must neither penetrate the interface nor spontaneously spread at the air/foaming solution interface. In other words, the oil droplets in the foaming solution do not wet the air/foaming solution interfaces: the immiscible liquid is non-wetting. Thus, $E=\gamma_{\mathrm{aw}}+\gamma_{\mathrm{ow}}-\gamma_{\mathrm{ao}}$ and $S=\gamma_{\mathrm{aw}}-\gamma_{\mathrm{ow}}-\gamma_{\mathrm{ao}}$, the entry and spreading coefficients defined using $\gamma_{a w}, \gamma_{o w}$ and $\gamma_{a o}$ the air/foaming solution, oil/foaming solution and oil/air surface tensions, must both be negative [20,22]. With our foaming solution and organic oils, such as olive oil or sunflower oil, both $E$ and $S$ exhibit negative values, as shown in Table I. The viscosity $\eta$ and the density $\rho$ of the two oils are also displayed in Table I.

To generate the foam, a slow flow of gaseous nitrogen is blown through a syringe needle into a $27 \mathrm{~cm}$-high rectangular column with a $6 \mathrm{~cm}$-wide square base filled with the foaming solution. We use different syringe needles to obtain monodisperse foams with an average radius $R$ of $0.5 \mathrm{~mm}, 1.8 \mathrm{~mm}$ or $2.5 \mathrm{~mm}$. Besides, the 5 -cm high upper part of the column is removable. Thus, we can extract at the top of the column a foam sample for which the volume $\Omega$ and the mass $m$ are known and measured by using a precise weighing scale. We deduce $\epsilon$ the liquid fraction of the sample from $\epsilon=m / \Omega \rho$ and typically $0.7 .10^{-4}<\epsilon<1.2 .10^{-3}$. With this set-up, we can therefore vary independently $\epsilon$ and $R$ and thus obtain curvature 


\begin{tabular}{|c|c|c|c|c|c|c|c|}
\hline Organic phase & $\gamma_{\text {aw }}$ & $\gamma_{\text {ao }}$ & $\gamma_{\text {ow }}$ & $\eta$ & $\rho$ & $E$ & $S$ \\
\hline Sunflower oil & 23.7 & 32.5 & 4.5 & 55 & 0.92 & -4 & -13 \\
Olive oil & 23.7 & 30.8 & 3 & 61 & 0.92 & -4 & -10 \\
\hline
\end{tabular}

TABLE I.

Characteristics of the oils used for imbibition with the foaming solution described in [20]. $\gamma_{\text {aw }}$,

$\gamma_{\mathrm{ow}}$ and $\gamma_{\mathrm{ao}}$ are the air-foaming solution, oil-foaming solution and oil-air surface tensions in $\mathrm{mN} / \mathrm{m}, \eta$ is the dynamic oil viscosity in mPa.s, $\rho$ is the density in $\mathrm{kg} . \mathrm{m}^{-3}$. $E$ and $S$ are the entry and spreading coefficient in $\mathrm{mN} / \mathrm{m}$.

radii of the Plateau border, noted $r_{F}$, between 15 and $85 \mu \mathrm{m}$ (see Figure 1-c)). Indeed, in the limit where $\epsilon<10^{-2}$, most of the liquid is comprised within the Plateau borders and $r_{F}=\sqrt{3} \sqrt{\epsilon} R$ from volume conservation $[23,24]$.

Given that the contrast of optical indexes between the foam and the liquid is small or null, we add a small quantity of fluorescent dyes to the liquid at a concentration of $10^{-2}$ g/g (fluorescein from Sigma-Aldrich for aqueous solutions and Yellow Black from Rohm and Haas for organic oils). Those fluorescent markers, which are trapped in the liquid, provide a means of visualizing the swollen part of the foam [19]. With this set-up and under illumination with excitation at $488 \mathrm{~nm}$, the liquid is luminous while the aqueous foam - only constituted of air and foaming solution - is not visible. The fluorescence intensity is followed with a camera recording at typically 10 frames per second.

Figure 1-d) illustrates such an experiment where a dry dark foam is put into contact with a large $20 \mu \mathrm{L}$ fluorescent oil drop sitting on a solid surface. We first observe a lateral spreading of the oil drop, squeezed between the solid surface and the foam. Yet, after ten seconds, oil wicks into the foam, revealing the foam architecture and forming a complex oilladen foam structure. After 5 minutes, oil has risen in the foam up to $h=10 \mathrm{~mm}$. The foam film withstands without any problem the oil invasion: we barely observe any film break-up during the whole process. This confirms that the imbibition only occurs within the Plateau borders and the nodes and does not interfere with the fragile structure of the foam films as previously observed at the scale of a single Plateau border [17]. Yet, the structure of this oil-laden foam is not frozen: elementary topological changes known as T1 are frequently observed due to liquid redistribution[19]. 

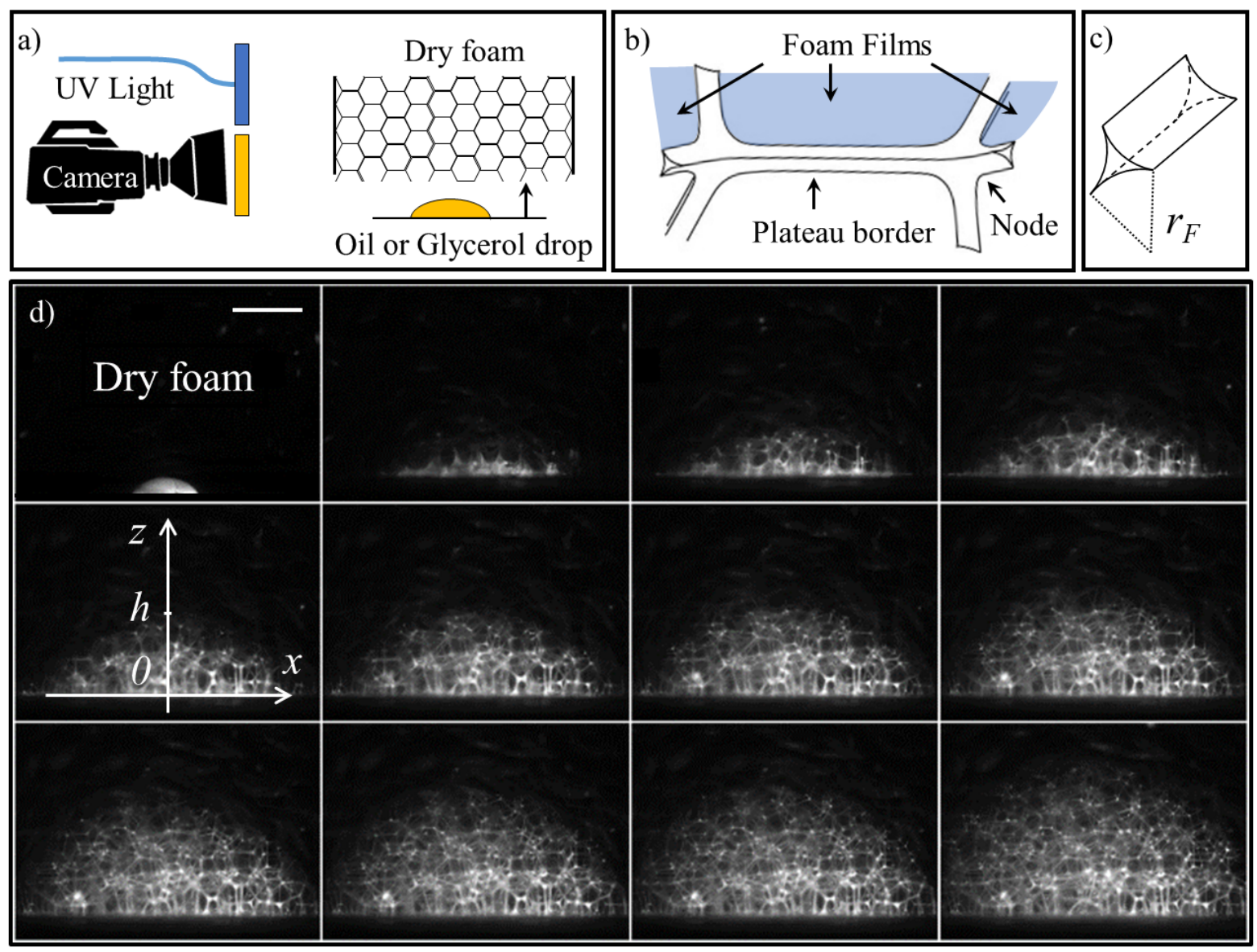

FIG. 1. a) Experimental set-up. b) Geometrical elements of foams: Nodes, Plateau borders and foam films. c) Slender Plateau border with a radius of curvature $r_{F}$. d) Capillary rise of oil in a dry aqueous foam. The olive oil drop appears white thanks to a fluorescent dye and invades the network of Plateau borders. Time interval between each frame is $67 \mathrm{~s}$. The scale bar is $3 \mathrm{~mm}$.

Our fluorescent set-up allows us to follow $h$, the upper boundary of the imbibition front in the foam as defined in the fifth frame of Figure 1-d). Figure 2 shows $h$ as a function of time $t$, when a $20 \mu \mathrm{L}$ olive oil drop is put into contact with foams at various liquid fractions. As classically observed in porous media, the early times dynamics is fast. Moreover, here, the drier the foam, the faster the early-time dynamics, a feature which underlines the importance of the liquid fraction of the foam prior to imbibition. When plotted in log-log scale, those curves do not exhibit a well-defined power law as can be seen on the inset of Figure 2, where two straight lines corresponding to the $t^{1 / 2}$ early and $t^{1 / 4}$ late-time dynamics are drawn. We also emphasize that for the driest foam, the front velocity is small: typically, 


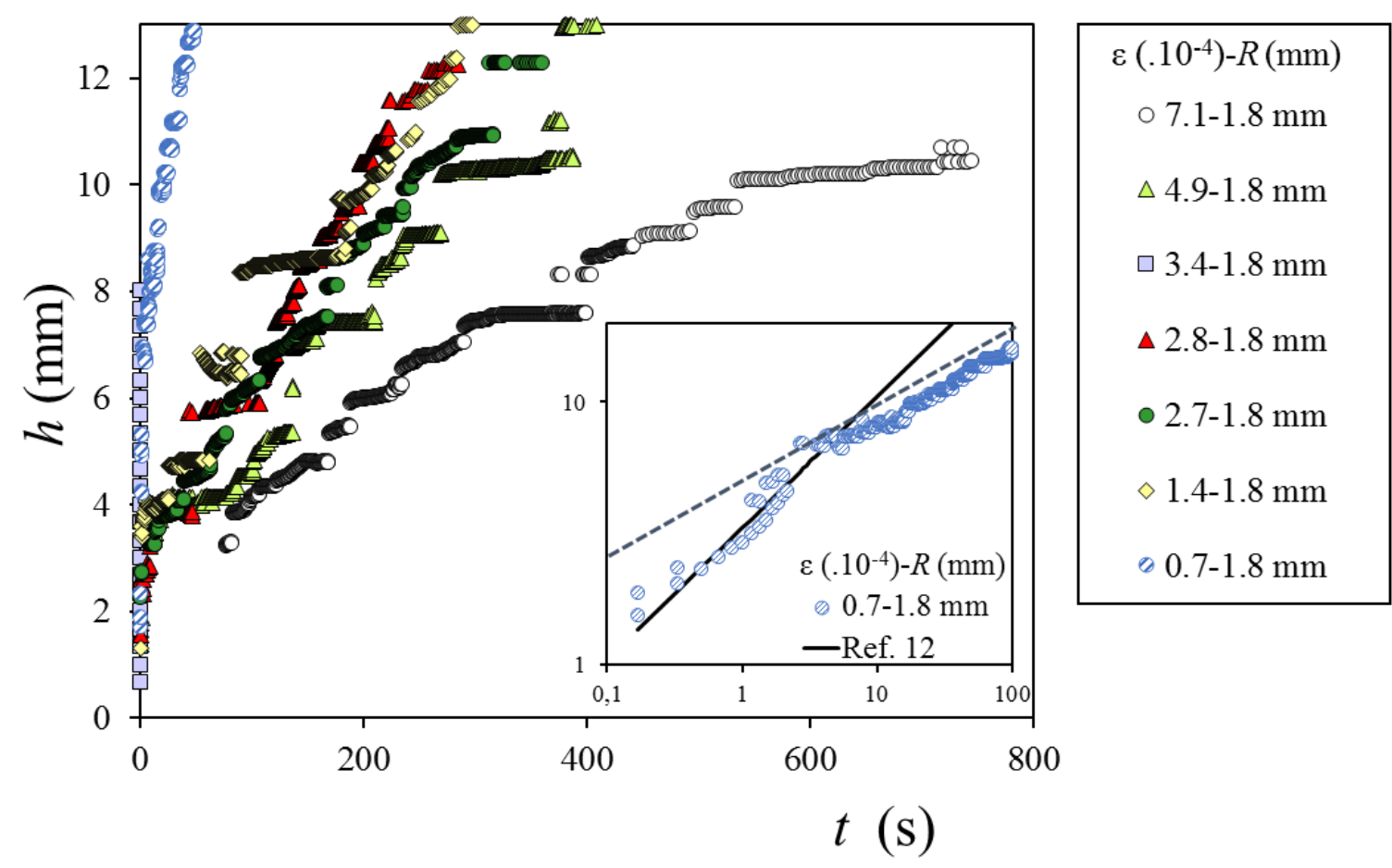

FIG. 2. Height of the rising front for olive oil as a function of time for different initial liquid fractions. Inset: $\log -\log$ scale of the data corresponding to $\epsilon=0.7 \cdot 10^{-4}$. The plain line corresponds to the $t^{1 / 2}$ dynamics with the diffusive coefficient given in [12], while the dashed line illustrates the $t^{1 / 4}$ dynamics.

$\dot{h} \sim 0.2 \mathrm{~mm} / \mathrm{s}$ at $t=10 \mathrm{~s}$. For a centimeter length scale, the Reynolds number is around $R e=\rho \dot{h} L / \eta \sim 10^{-8}<<1$. Note that the imbibition of glycerol or sunflower oil exhibits the same trends.

The rigid boundary conditions provided by the foaming solution and the small value of $R e$ suggest that the fluid motion in the foam network corresponds to a Stokes flow where the capillary driving force is balanced with the viscous dissipation. In the limit where $\gamma_{\mathrm{ow}} / \gamma_{\mathrm{aw}}<1$, recent experimental works at the scale of a single horizontal Plateau border have revealed the following features [17, 25]. On one hand, the driving capillary pressure that sustains the imbibition in the Plateau border is set by $\gamma_{a w} / r_{F}$, where $r_{F}$ is the curvature radius of the Plateau border prior to imbibition. On the other hand, the bulk viscous dissipation only occurs in the oil phase and scales as $\eta \dot{z} / r^{2}(z, t)$, where $r^{2}(z, t)$ is 


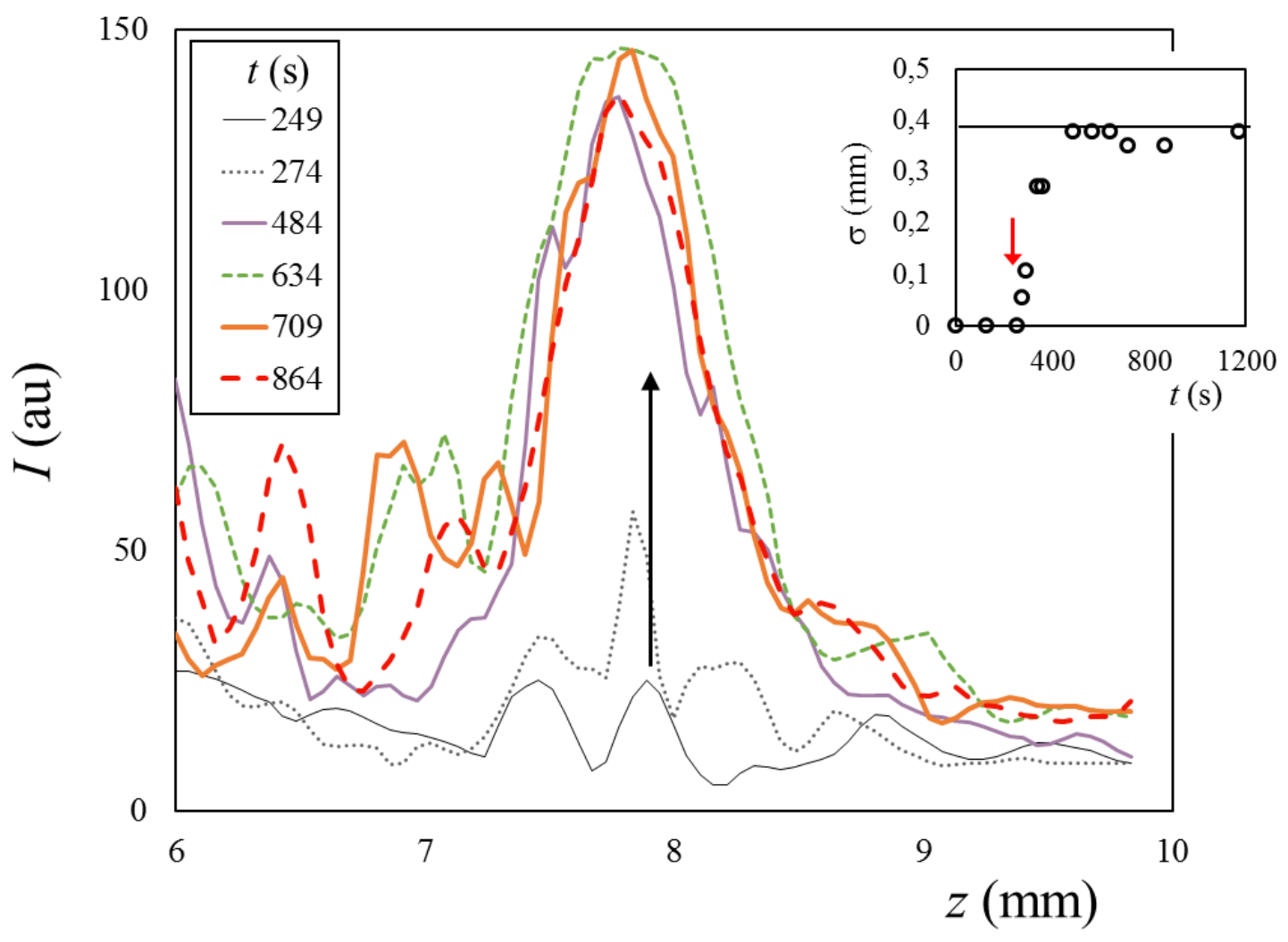

FIG. 3. Typical evolution of the fluorescence intensity of a single node during sunflower oil imbibition as a function of the node's vertical dimension. Initially, the node is located at $x=1 \mathrm{~mm}$ and $z=7.9 \mathrm{~mm}$. The intensity of fluorescence is in arbitrary units and the different curves correspond to fluorescence intensity measurements taken at different times. The black arrow indicates the evolution of time. Inset: Width of the peak of intensity (defined at half of the maximum intensity) as a function of time. The red arrow highlights the time at which the oil front has reached the node.

proportional to the swollen Plateau border cross section. In reference [17], the experiments were either driven at constant flow rate or constant volume of oil, thus $r(z, t)$ was deduced from mass conservation [26], while here imbibition occurs from a large reservoir. Thus, to gain quantitative understanding of the spatial and temporal evolution of $r$, we take advantage of the fluorescence intensity of our images. In Figure 3, we report the spatial evolution of the fluorescence intensity of a single node located at $x=1 \mathrm{~mm}$ and $z=7.9 \mathrm{~mm}$ for different times. The oil front reaches the height $z=7.9 \mathrm{~mm}$ at $t=270 \mathrm{~s}$, thus the signal prior to $t=270 \mathrm{~s}$ corresponds to noise fluctuation. At $t=274 \mathrm{~s}$, a peak in the fluorescence intensity is observed. The intensity of this peak grows until reaching a steady shape observed $100 \mathrm{~s}$ 
after the front has gone through the node, as can be seen in the inset of Figure 3 where a saturation of the peak's width is observed after $t=400 \mathrm{~s}$. This suggests that for the foam below the oil front, the distribution of oil quickly reaches a steady-state profile. This steadystate profile must match the equilibrium profile given by a balance between capillarity and gravity $[23,24]$. At late time and for $z<<h, r$ does not depend anymore on $t$ and:

$$
r(z, t)=r(z) \sim \frac{R}{1+\alpha z}
$$

where $\alpha=\frac{\rho g R}{\gamma_{\text {aw }}+\gamma_{\mathrm{ow}}} \sim \frac{\rho g R}{\gamma_{\mathrm{aw}}}$, has the dimension of an inversed length. This classic profile illustrates the complex multi-scale geometry of foam under gravity: for $z<1 / \alpha$, the curvature is only set by the bubble size $r \sim R$, while for $z>1 / \alpha, r \sim R / \alpha z$. To describe the imbibition dynamics within the aqueous foam, we consider the flow throughout the slender Plateau borders below the front $[23,24]$ and use the Hagen-Poiseuille law:

$$
v=-\frac{K_{c} \delta r(z)^{2}}{\eta} \frac{d P}{d z}
$$

where $r(z)$ is given by Eq. 1 and $v, \eta, K_{c}$ represent the average velocity of the liquid, the liquid dynamic viscosity and the permeability coefficient for infinite slender channels, $K_{c}$ $=1 / 50 . \quad \delta=0.16$ is a numerical factor linking $r$ and $A$, the cross-section of a Plateau border so that $A=\delta r^{2}$ [27-29]. Then, we write mass conservation, which yields $v(z)=v(h) r^{2}(h) / r^{2}(z)=\dot{h} r^{2}(h) / r^{2}(z)$. Integrating Eq. 2 between $0<z<h$ with the boundary conditions $p(z=0)=p_{o}$ and $p(h)=p_{o}-\gamma_{\text {aw }} / r_{F}$ and using Eq. 1 gives:

$$
\dot{h}\left((1+\alpha h)^{3}-\frac{1}{(1+\alpha h)^{2}}\right)=\frac{\alpha \delta \gamma_{\mathrm{aw}} R^{2}}{10 \eta r_{F}}
$$

Eq. 3 is then integrated with the condition $h(t=0)=0$ :

$$
(1+\alpha h)^{4}+\frac{4}{1+\alpha h}-5=\frac{2 \delta}{5 \sqrt{3}} \frac{\alpha^{2} R \gamma_{\mathrm{aw}}}{\sqrt{\epsilon} \eta} t
$$

At late times or for $\alpha h>>1$, Eq. 4 predicts an evolution of the rising front in $t^{1 / 4}$, while for $\alpha h<<1$, a second-order limited expansion of Eq. 4 gives $h^{2} \sim R \gamma_{\text {aw }} t / \sqrt{\epsilon} \eta$, thus suggesting an early time dynamics of imbibition in $t^{1 / 2}$. These regimes intersect for $h \sim 1 / \alpha$, the typical height for which the curvature radius of the foam changes from $R$ to $R / \alpha h$. Moreover, Eq. 4 suggests a collapse of the data using the dimensionless variables $\alpha h$ and $t^{*}=\frac{\alpha^{2} R \gamma_{\text {aw }}}{\sqrt{\epsilon} \eta} t$, which is in very good agreement with our experimental results obtained for 


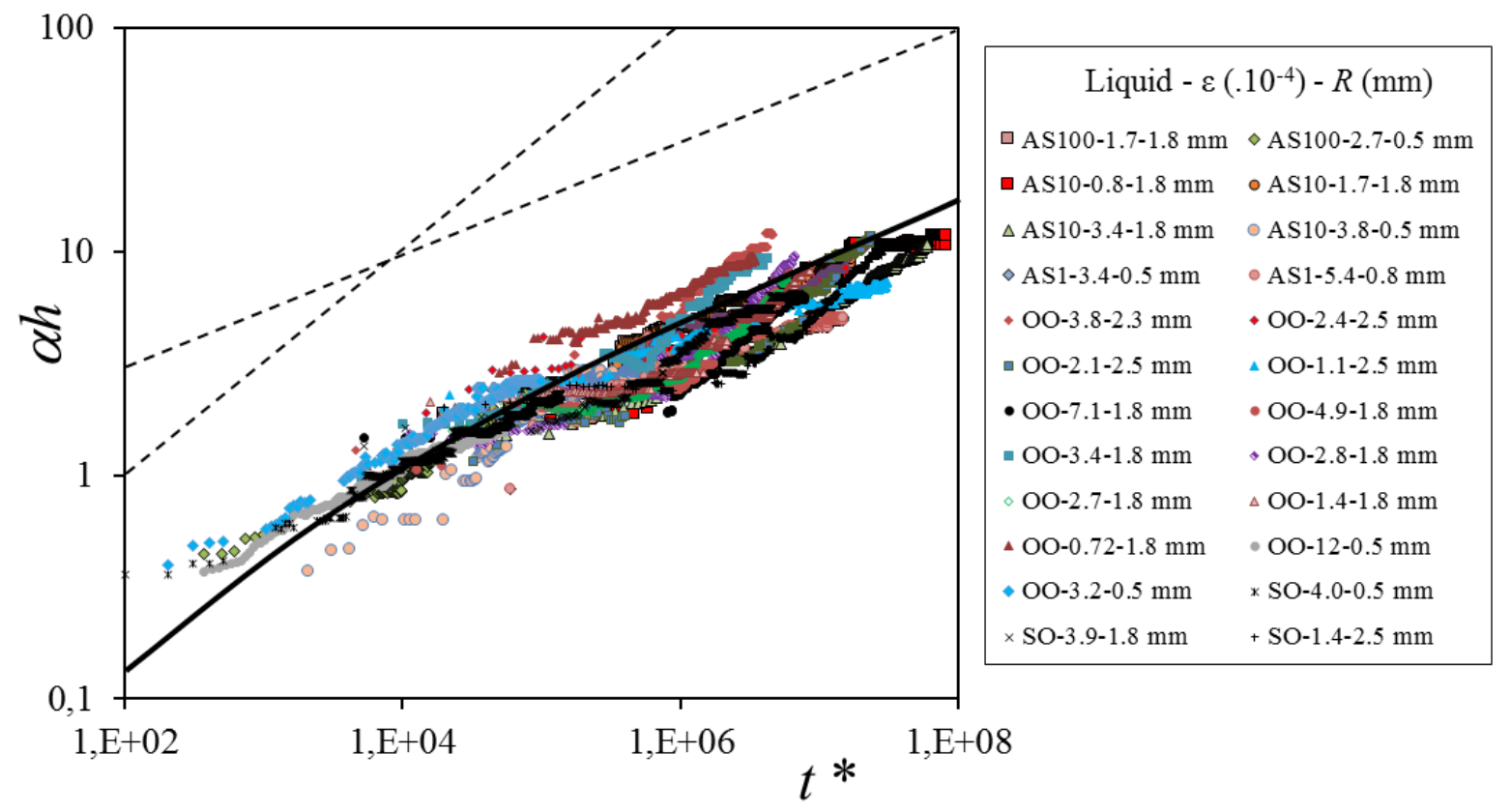

FIG. 4. Normalized imbibition front $\alpha h$ as a function of normalized time $t^{*}$ in log-log plot. In the legend, AS1, AS10 and AS100 respectively stand for Aqueous Solution with $\eta=1.4,10$ or $100 \mathrm{mPa} . \mathrm{s}$, while OO and SO stands for Olive Oil and Sunflower Oil. The black line corresponds to $(1+\alpha h)^{4}+\frac{4}{1+\alpha h}-5=b \frac{2 \delta}{5 \sqrt{3}} t^{*}$ with $b \sim 0.1$, while the two dashed lines to the power laws: $t^{1 / 2}$ at early times and $t^{1 / 4}$ at late times.

three different liquids, either organic or aqueous, and foams with various bubble diameters and initial liquid fractions, as can be seen in Figure 4. Furthermore, the data are well fitted by $(1+\alpha h)^{4}+\frac{4}{1+\alpha h}-5=b \frac{2 \delta}{5 \sqrt{3}} t^{*}$, with a coefficient $b$ deduced from the experiments of $b \sim 0.1$.

Foam drainage theory provides different scaling laws for foam imbibition, which can be confronted to our results. In particular, the diffusive regime observed under microgravity conditions $[10,11]$ is in very good agreement with our data as can be seen in the inset of figure 2, thus underlining the negligible role of gravity when the foam is wet at the bottom. At late times, analytical and numerical calculations for the imbibition of infinitely dry foams on Earth 
gives [13]:

$$
h \sim\left(\frac{\gamma_{\mathrm{aw}}^{2}}{\eta \rho g} t\right)^{1 / 3}
$$

The scatter in the data makes it difficult to distinguish between the different temporal regimes of equations 4 and 5. Yet, contrary to equation 4, equation 5 neither captures the influence of $\epsilon$ nor $R$ that is observed in the experiments. This can be understood recalling that Equation 5 has been derived i) solving the Partial Differential Equation (PDE) ruling the evolution of $\left.r_{(} z, t\right)$ ii) assuming a foam with an initial liquid fraction equal to zero and iii) seeking for self-similar solutions of the PDE. This procedure is particularly useful to describe the asymptotic behavior of solutions in the limit where these no longer depend on the detail of the initial and boundary conditions. However, in our experiment, the foam sample is only 5-cm high, not infinitely dry and the time-scale is limited. Thus, the initial and boundary conditions can not be neglected. We therefore never observe any convergence of the dynamics towards the $t^{1 / 3}$ self-similar regime.

We highlight the ability of dry liquid foams to displace and drive upward miscible or immiscible liquids on Earth. This remarkable feature is of considerable interest in various industries such as soil remediation, detergency, shampoo industry, enhanced oil recovery and dismantling of nuclear power plants, where foams are used for their low density, non-newtonian rheology and the low quantity of liquid waste they generate. Considering steady-state solutions and rigid interfaces in the framework of porous media, we provide a physical picture for this imbibition dynamics, which captures well our experimental observations concerning the bubble radius $R$, the initial liquid fraction $\epsilon$ and the long-term dynamics. Yet, integrating this physical picture in the framework of the foam drainage equation, which has proven successful for both mobile and rigid interfaces remains to be done.

We thank O. Pitois, M. Adler, A. Delbos, F. Rouyer, A.L. Biance, I. Cantat, Y. Peysson, F. Chevoir and E. Reyssat for fruitful discussions. Samples of surfactants were kindly provided by M. Pepin from Stepan Europe and R. Roth from Evonik. We gratefully acknowledge financial support from Agence Nationale de la Recherche (ANR- 11-JS09-012-WOLF). 
[1] R. Lucas, Kolloid Z. 23, 15 (1918).

[2] E. W. Washburn, Phys. Rev. 17, 273 (1921).

[3] A. Marmur and R. Cohen, J. Coll. Int. Sci. 189, 299 (1997).

[4] M. Lago and M. Araujo, J. Coll. Int. Sci. 234, 35 (2001).

[5] G. Tampy, W. Cen, M. Prudich, and R. Savage, ENERGY \& FUELS 2, 782 (1988).

[6] T. Delker, D. Pengra, and P. Wong, Phys. Rev. Lett. 76, 2902 (1996).

[7] J. I. Siddique, D. M. Anderson, and A. Bondarev, Phys. Fluids 21, 013106 (2009).

[8] M. Reyssat, L. Courbin, E. Reyssat, and H. A. Stone, J. Fluid Mech. 615, 335 (2008).

[9] C. Duprat, J. M. Aristoff, and H. A. Stone, J. Fluid Mech. 679, 641 (2011).

[10] A. Saint-Jalmes, S. Marze, H. Ritacco, D. Langevin, S. Bail, J. Dubail, L. Guingot, G. Roux, P. Sung, and L. Tosini, Phys. Rev. Lett. 98, 058303 (2007).

[11] H. Caps, S. Cox, H. Decauwer, D. Weaire, and N. Vandewalle, COLSUA 261, 131 (2005).

[12] S. Cox, D. Weaire, and G. Verbist, Eur. Phys. J. B 40, 119 (2004).

[13] S. Koehler, H. Stone, M. Brenner, and J. Eggers, Phys. Rev. E 58, 2097 (1998).

[14] A. Ponomarenko, D. Quere, and C. Clanet, J. Fluid Mech. 666, 146 (2011).

[15] T. Cambau, J. Bico, and E. Reyssat, Europhys. Lett. 96, 24001 (2011).

[16] L. Tang and Y. Tang, J. Phys. II 4, 881 (1994).

[17] K. Piroird and E. Lorenceau, Phys. Rev. Lett. 111, 234503 (2013).

[18] J. Sonoda, T. Sakai, and Y. Inomata, J. Phys. Chem. B 118, 9438 (2014).

[19] K. Piroird, E. Lorenceau, and A.-L. Biance, Soft Matter 10, 7061 (2014).

[20] E. Basheva, D. Ganchev, N. Denkov, K. Kasuga, N. Satoh, and K. Tsujii, Langmuir 16, 1000 (2000).

[21] K. Golemanov, N. D. Denkov, S. Tcholakova, M. Vethamuthu, and A. Lips, Langmuir 24, 9956 (2008).

[22] P. Garrett, Defoaming: theory and industrial applications (CRC, 1992).

[23] I. Cantat, S. Cohen-Addad, F. Elias, F. Graner, R. Höhler, O. Pitois, F. Rouyer, and A. SaintJalmes, Foams : Structure and Dynamics (Oxford University Press, 2013).

[24] D. Weaire and S. Hutzler, The Physics of Foams (Oxford University Press, 1999).

[25] A. Cohen, N. Fraysse, J. Rajchenbach, M. Argentina, Y. Bouret, and C. Raufaste, Phys. Rev. 
Lett. 112, 218303 (2014).

[26] P. Warren, Phys. Rev. E 69, 041601 (2004).

[27] S. Cohen-Addad, R. Hohler, and O. Pitois, Ann. Rev. Fluid Mech. 45, 241 (2013).

[28] A. Nguyen, J. Coll. Int. Sci. 249, 194 (2002).

[29] S. Koehler, S. Hilgenfeldt, and H. Stone, J. Coll. Int. Sci. 276, 420 (2004). 